\title{
Erratum to: Nonuse of dental service by schoolchildren in Southern Brazil: impact of socioeconomics, behavioral and clinical factors
}

\author{
Vanessa Polina Pereira da Costa • Marília Leão Goettems • \\ Luísa Jardim Corrêa de Oliveira - Sandra Beatriz Chaves Tarquinio • \\ Dione Dias Torriani · Marcos Britto Correa • \\ Flávio Fernando Demarco
}

Published online: 26 July 2015

(C) Swiss School of Public Health 2015

\section{Erratum to: Int J Public Health (2015) 60:411-416 DOI 10.1007/s00038-015-0670-2}

Unfortunately, some errors occurred in the original publication of the article.

The last sentence in the second paragraph of the "Results" section (p. 413) should read as "After adjustments, children with lower maternal schooling and lower income, those enrolled in public schools, those with DMFT $=0$, those with dental fear, those living in more crowded households, and those initiating tooth-brushing in later ages were associated with the lack of use of dental service."
The third sentence in the second paragraph of the "Discussion" section (p. 413) should read as "Our findings show that children from mothers with lower schooling use dental services $46 \%$ less often than children from more educated mothers, and children from families with lower income use dental services $81 \%$ less often than children from families with high income."

Further, in Table 2, the entries of the last four columns have been assigned to wrong column heads. The corrected Table 2 is given in the following page.

The online version of the original article can be found under doi:10. 1007/s00038-015-0670-2.

V. P. P. da Costa - M. L. Goettems - L. J. C. de Oliveira .

S. B. C. Tarquinio - D. D. Torriani - M. B. Correa .

F. F. Demarco $(\square)$

Post-Graduate Program in Dentistry, Federal University of

Pelotas, Pelotas, RS, Brazil

e-mail: ffdemarco@gmail.com

\section{F. F. Demarco}

Post-Graduate Program in Epidemiology, Federal University of

Pelotas, Pelotas, RS, Brazil 
Table 2 Description, crude and adjusted analysis of children who had never used dental services. Pelotas, Brazil, $2010(n=291)$

\begin{tabular}{|c|c|c|c|c|c|c|}
\hline \multirow[t]{2}{*}{ Variable } & \multicolumn{2}{|c|}{ Never visited a dentist } & \multirow[t]{2}{*}{ RP crude $(95 \% \mathrm{CI})$} & \multirow[t]{2}{*}{$P$} & \multirow[t]{2}{*}{$\mathrm{RP}$ adjusted $(95 \% \mathrm{CI})$} & \multirow[t]{2}{*}{$P$} \\
\hline & $n$ & $\%$ & & & & \\
\hline \multicolumn{7}{|l|}{ Sex } \\
\hline Male & 127 & 22.56 & 1.00 & & 1.00 & 0.295 \\
\hline Female & 164 & 25.99 & $1.15(0.94-1.41)$ & 0.169 & $1.12(0.91-1.38)$ & \\
\hline \multicolumn{7}{|l|}{ Age (years) } \\
\hline 8 & 48 & 26.67 & 1.00 & 0.151 & 1.00 & 0.092 \\
\hline 9 & 79 & 25.57 & $0.96(0.70-1.30)$ & & $0.94(0.67-1.33)$ & \\
\hline 10 & 77 & 26.46 & $0.99(0.73-1.35)$ & & $1.06(0.76-1.47)$ & \\
\hline 11 & 49 & 19.37 & $0.73(0.51-1.03)$ & & $0.78(0.54-1.13)$ & \\
\hline 12 & 38 & 23.60 & $0.89(0.61-1.28)$ & & $0.77(0.51-1.56)$ & \\
\hline \multicolumn{7}{|l|}{$\begin{array}{l}\text { Family monthly } \\
\text { income (quartiles) }\end{array}$} \\
\hline 4 & 20 & 8.16 & 1.00 & $<0.001$ & 1.00 & 0.004 \\
\hline 3 & 58 & 21.40 & $2.62(1.63-4.23)$ & & $1.39(0.85-2.29)$ & \\
\hline 2 & 77 & 32.08 & $3.93(2.48-6.22)$ & & $1.76(1.08-2.85)$ & \\
\hline 1 & 102 & 36.56 & $4.48(2.86-7.00)$ & & $1.81(1.12-2.93)$ & \\
\hline \multicolumn{7}{|l|}{ Maternal schooling } \\
\hline$>8$ years & 127 & 16.98 & 1.00 & $\leq 0.001$ & 1.00 & 0.001 \\
\hline$\leq 8$ years & 159 & 37.41 & $2.20(1.80-2.69)$ & & $1.46(1.17-1.82)$ & \\
\hline \multicolumn{7}{|l|}{ Type of school } \\
\hline Private & 10 & 3.97 & 1.00 & $\leq 0.001$ & 1.00 & $\leq 0.001$ \\
\hline Public & 281 & 29.83 & $7.52(4.06-13.91)$ & & $8.32(3.10-22.32)$ & \\
\hline \multicolumn{7}{|l|}{ DMFT } \\
\hline$\geq 1$ & 75 & 19.58 & 1.00 & 0.23 & 1.00 & $\leq 0.001$ \\
\hline 0 & 215 & 26.54 & $1.35(0.87-1.03)$ & & $1.70(1.34-2.16)$ & \\
\hline \multicolumn{7}{|l|}{ Dental fear } \\
\hline No & 176 & 19.71 & 1.00 & $\leq 0.01$ & 1.00 & $\leq 0.01$ \\
\hline Yes & 114 & 39.04 & $1.98(1.63-2.41)$ & & $1.62(1.30-2.01)$ & \\
\hline \multicolumn{7}{|l|}{ Family structure } \\
\hline Nuclear & 170 & 23.35 & 1.00 & 0.275 & & \\
\hline Non-nuclear & 121 & 26.13 & $1.12(0.91-1.37)$ & & & \\
\hline \multicolumn{7}{|l|}{ Household crowding } \\
\hline $0-4$ & 141 & 19.18 & 1.00 & $\leq 0.01$ & 1.00 & 0.003 \\
\hline 5 & 53 & 22.94 & $1.20(0.90-1.58)$ & 0.34 & $1.03(0.76-1.39)$ & \\
\hline$\geq 6$ & 96 & 42.67 & $2.22(1.80-2.75)$ & 0.04 & $1.55(1.23-1.97)$ & \\
\hline \multicolumn{7}{|l|}{ Started brushing } \\
\hline With eruption & 62 & 14.90 & 1.00 & $\leq 0.01$ & 1.00 & 0.047 \\
\hline With 2 years or more & 217 & 28.86 & $1.94(1.50-2.50)$ & & $1.30(1.00-1.67)$ & \\
\hline \multicolumn{7}{|l|}{ Self-perceived oral health } \\
\hline Excellent-Good & 81 & 20.30 & 1.00 & 0.023 & 1.00 & 0.438 \\
\hline Fair-poor & 208 & 26.39 & $1.30(1.04-1.63)$ & & $0.91(0.72-1.15)$ & \\
\hline
\end{tabular}

